\title{
Diffusion of liquid domains in lipid bilayer membranes
}

\author{
Pietro Cicuta, ${ }^{1}$ Sarah L. Keller, ${ }^{2}$ and Sarah L. Veatch ${ }^{3, *}$ \\ ${ }^{1}$ Cavendish Laboratory and Nanoscience Center, University of Cambridge, Cambridge CB3 OHE, U.K. \\ ${ }^{2}$ Departments of Chemistry and Physics, University of Washington, Seattle, WA 98195-1700, USA \\ ${ }^{3}$ Department of Microbiology and Immunology, University of British Columbia, Vancouver, BC, V6T1Z4, Canada
}

\begin{abstract}
We report diffusion coefficients of micron-scale liquid domains in giant unilamellar vesicles of phospholipids and cholesterol. The trajectory of each domain is tracked, and the mean square displacement grows linearly in time as expected for Brownian motion. We study domain diffusion as a function of composition and temperature, and measure how diffusion depends on domain size. We find mechanisms of domain diffusion which are consistent with membrane-dominated drag in viscous $\mathrm{L}_{o}$ phases [P.G. Saffman and M. Delbrück, PNAS 72, 3111 (1975)], and bulk-dominated drag for less viscous $\mathrm{L}_{\alpha}$ phases [B.D.Hughes et al., J. Fluid Mech. 110, 349 (1981)]. Where applicable, we obtain the membrane viscosity and report activation energies of diffusion.
\end{abstract}

PACS numbers: 68.35.Fx, 68.55.Ac, 87.16.Dg

Diffusion of domains within cell membranes is a highly relevant biophysical problem. The presence of lipid domains, including rafts, can affect both short-range (intradomain) and long-range (inter-domain) diffusion of membrane components [1, 2]. Diffusion has been observed in live cell membranes 3 , 4] , although interpreting results from these complex systems can be challenging.

Even in simple model systems, deciphering the diffusion of membrane inclusions is a long-standing and difficult hydrodynamic problem [5, 6, 7]. Objects that diffuse in the membrane plane range from small peptides and individual lipids to large inclusions like protein aggregates and lipid domains. The first challenge is that, unlike in three dimensional (3D) diffusion, the size of the diffusing object is not the only length-scale that enters into the problem. For example, the membrane has finite thickness, a finite surface area, and often a nonzero curvature. Secondly, the membrane is composed of macromolecules, which limits continuum approaches to large objects. Lastly, the membrane and its surroundings have different viscosities. In complex biological membranes, additional length-scales may be important, such as the distance between membrane proteins [8] or the size of corrals created by the actin cytoskeleton [3].

In this Letter, we directly measure diffusion of liquid domains in giant unilamellar vesicles (GUVs) of radius $\simeq 20 \mu \mathrm{m}$ as in Figure 1. These domains are micron-scale, circular, span the lipid bilayer, and undergo Brownian motion. By measuring diffusion of bilayer domains over a wide parameter range of more than one decade in domain radii and three decades in $2 \mathrm{D}$ membrane viscosities, we probe the two limiting models of Saffman-Delbrück [5] and Hughes et al. [6]. We find a cross-over between the two models which would not have been predicted from previous monolayer results [11]. In the cases where our data are well fit by the Saffman-Delbrück equation, we are able to extract viscosities of lipid phases and diffusion activation energies.

Domains move in a background phase with two dimen- sional (2D) membrane viscosity $\left(\eta^{\prime \prime}\right)$. The diffusion coefficient of a membrane inclusion was originally described by Saffman and Delbrück [5]:

$$
D(r)=\frac{k_{B} T}{4 \pi \eta^{\prime \prime}}\left[\log \left(\frac{\eta^{\prime \prime}}{\eta_{w}} \frac{1}{r}\right)-\gamma+\frac{1}{2}\right]
$$

where $r$ is the radius of the inclusion, $\gamma=0.5772$ and we have chosen boundary conditions appropriate for liquid domains in a liquid membrane to yield the factor of $1 / 2$. A key parameter in the hydrodynamics of this system is the lengthscale $\lambda_{0}$ defined by the ratio of the membrane $\eta^{\prime \prime}$ to the 3D bulk viscosity of water $\left(\eta_{w}\right)$ such that $\lambda_{0}=$ $\eta^{\prime \prime} / \eta_{w}$. Eq. 1 is expected to hold for $r<\lambda_{0}$, i.e. small domains and/or large membrane viscosity. Later work confirmed this calculation and derived a result for the

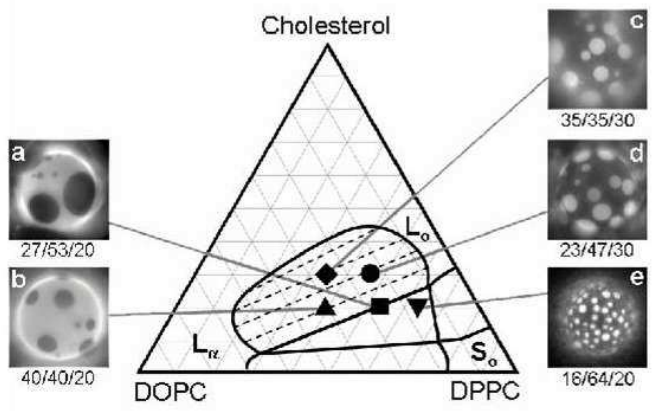

FIG. 1: Fluorescence microscopy phase diagram of $\mathrm{DOPC} / \mathrm{DPPC} /$ cholesterol and vesicle images at $20^{\circ} \mathrm{C}$. Semiquantitative dashed tie-lines cross the $\mathrm{L}_{\alpha}-\mathrm{L}_{o}$ coexistence region [9]. Some vesicles studied have a continuous $\mathrm{L}_{\alpha}$ (bright) phase (a-b) whereas others have a continuous $\mathrm{L}_{o}$ (dark) phase (c-d). One composition (e) has a continuous dark $\mathrm{L}_{o}$ phase which may contain both $\mathrm{L}_{o}$ and gel $\left(\mathrm{S}_{o}\right)$ phase lipids [10]. Vesicle compositions are shown as mol\% DOPC/DPPC/Chol. 


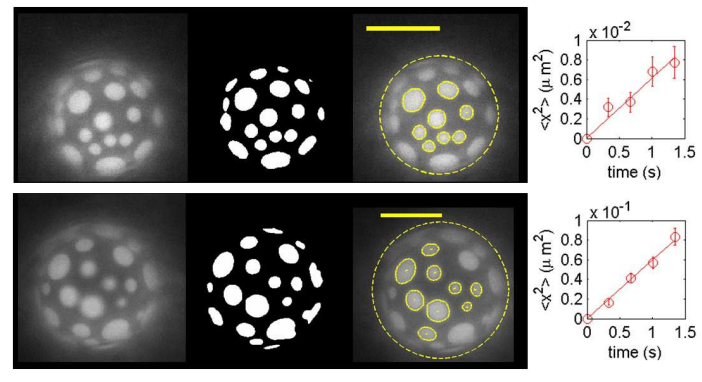

FIG. 2: Greyscale fluorescence images (left) are filtered and thresholded (middle). White regions are identified as domains. Those within a specified size range, ellipticity and distance from the edge are retained. Circles (right image) identify those domains successfully identified through 5 successive frames. Mean square displacement data for domains with radii of $1-1.5 \mu \mathrm{m}$ are shown at right. Both vesicles have composition 1:2 DOPC:DPPC $+30 \% \mathrm{Chol}, \mathrm{T}=10^{\circ} \mathrm{C}$ (top) and $20^{\circ} \mathrm{C}$ (bottom). Note the factor of ten difference in diffusion coefficients. The scale bar is $40 \mu \mathrm{m}$.

opposite limit of $\lambda_{0}<r[\underline{6}]$ :

$$
D(r)=\frac{k_{B} T}{16 \eta_{w}} \frac{1}{r}
$$

It is important to notice that for $\lambda_{0}<r$ the diffusion coefficient is more strongly dependent on the inclusion's radius, but is independent of the membrane viscosity. This case was verified experimentally through observations of micron-scale domains in monolayers [11]. Other theoretical work has addressed different inclusion shapes as well as large domains 12 .

Spherical giant unilamellar vesicles (GUVs; $30-100 \mu \mathrm{m}$ diameter) are made by electroformation [13] of a ternary mixture of cholesterol with phospholipids of both high (DPPC; di(16:0)PC) and low (DOPC; di(18:1)PC) melting temperatures. Materials and methods have been described previously [10]. The vesicle membranes are initially uniform at high temperature, and phase separate into two liquid phases when vesicle suspensions are placed on a pre-cooled microscope stage. The less viscous $\mathrm{L}_{\alpha}$ phase is labeled by fluorescent dye (Texas Red-DPPE). The composition and viscosity of the two phases depend on the composition and temperature of the entire vesicle. With time, domains coalesce, allowing us to probe a range of domain sizes at constant temperature.

We probe five lipid compositions in the ternary system of DOPC/DPPC/Chol, as in Figure 1. In previous microscopy and ${ }^{2} \mathrm{H}$ NMR measurements, we established that vesicles with these five compositions separate into a liquid-ordered $\left(\mathrm{L}_{o}\right)$ phase rich in the saturated lipid DPPC and a liquid crystalline $\left(\mathrm{L}_{\alpha}\right)$ phase rich in the unsaturated lipid DOPC [10, 14]. Two of the five compositions contain a continuous bright $\mathrm{L}_{\alpha}$ phase (Figure [1a-b), and two contain a continuous dark $\mathrm{L}_{o}$ phase (Figure 1-d). One composition falls within a three phase region (Figure 1p.) The presence of three phases is clear in
${ }^{2} \mathrm{H}$ NMR experiments (manuscript in preparation), but is difficult to detect by microscopy. We probe the viscosity of the continuous phase by tracking domains of the minority phase.

Membrane domains are identified by an image processing program written in Matlab (Figure 2). A Gaussian filter is applied to images before thresholding, to identify features in the size range of domains. Almost no domains are lost by this algorithm. Domains are accepted if: a) the diameter falls between a minimum (2 pixel) and maximum value; $b$ ) the shape is circular, such that all points in the domain perimeter lie within $20 \%$ of the mean domain radius; and c) the center of mass lies within a circle defined by 0.8 of the vesicle radius. Since all domains are round (shape fluctuations are minimal), these criteria discriminate against occasional problems arising from image analysis filtering (for example, two domains very close to each other will not be accepted).

A separate program tracks domain trajectories with logic similar to existing codes, i.e. by matching a domain with the nearest feature in the next image [15]. Average diffusion is subtracted to yield unbiased domain motion. Matching generates no false positives but does not have a perfect success rate. We therefore divide each movie (typically 100 frames at $0.34 \mathrm{~s} /$ frame) into 20 sets of 5 frames over which most domains are tracked successfully. Domain size does not change over this period. The average of vertical and horizontal mean square displacements (MSD) is linear with time $t$ and fit to $\left\langle x^{2}\right\rangle=2 D(r) t$ as expected for diffusion. Over five frames, the MSD is $\leq 0.03 \mu \mathrm{m}^{2}$ which is much smaller than the particle separation, and we see no effect of domain packing.

Figure 3 shows diffusion coefficients as a function of domain size. The data has been culled to report only sets in which wide ranges of domain sizes are observed for any fixed temperature and composition. The dashed lines in Figure 3 show the asymptotic $1 / r$ behavior given in Eq. 2 . Within error, all data fall on or below this theoretical upper bound in diffusion coefficient. Eq. 22 is independent of membrane viscosity and holds when membrane viscosity is low, or domain radius is large i.e. when $\lambda_{0} \ll r[6]$. For the low viscosity $L_{\alpha}$ phase (e.g. Figure 3 d), it can be seen that the conditions of low membrane viscosity and large domain radius are met through most of the temperature range, because most data fall along the dashed line.

All data below the dashed line in Figure 3 correspond to membranes with high viscosity, notably $\mathrm{L}_{o}$ phases at low temperatures. We have chosen to fit our data to the Saffman-Delbrück equation, which should hold when membrane viscosity is high. It is clear that this set of data does not have a $D(r) \sim r^{-1}$ dependence, and we find instead reasonable fits to Eq. 1 with a single fitting parameter $\left(\eta^{\prime \prime}\right)$. Since our domain radii are limited to $\geq 0.5 \mu \mathrm{m}$ by our optical resolution and $\leq 10 \mu \mathrm{m}$ by our vesicle diameters, we cannot prove that Eq. 1 is the only 


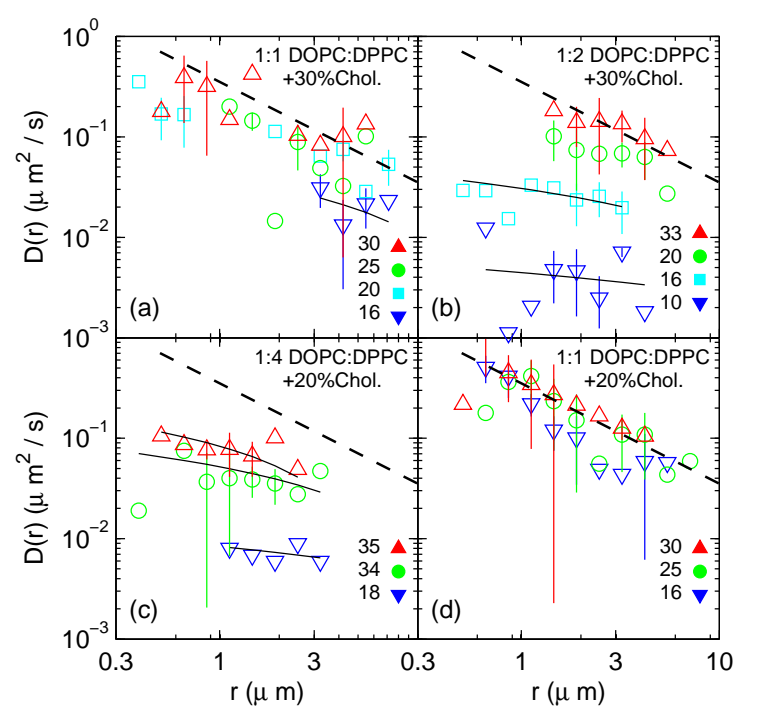

FIG. 3: Diffusion coefficients vary with domain radius. Solid lines show fits to a logarithmic dependence on domain size as in the Saffman-Delbrück Eq. 11 These fits have only one free parameter, as discussed in the text. Symbols identify increasing temperatures, recorded each plot. Dashed lines are fits to Eq. 4 with no free parameters. Error bars report standard deviations for sets with $\geq 3$ measurements.

expression that could fit our data. Nevertheless, fitting to Eq. 1 allows us to extract $\eta^{\prime \prime}$ for high viscosity membranes.

There have been multiple experiments designed to test the logarithmic form of Eq. 11 7, 11, 16], and its range of applicability is still controversial. For example, recent work asserts that individual proteins diffuse with a stronger size dependence, as $D(r) \sim r^{-1}$ [16], due to a break-down of the continuum approximation of the membrane for small inclusions [16, 17]. Here we explore domain radii well within the continuum limit $\left(r \gg r_{\text {single molecule }} \sim 0.5 \mathrm{~nm}\right)$.

Our results can be extrapolated to estimate the diffusion rate of raft domains in cell membranes. Lipid rafts are reported to have diameters of $10-100 \mathrm{~nm}$ 18]. This length-scale falls within the regime where the SaffmanDelbrück equation should apply. We calculate diffusion coefficients for $10-100 \mathrm{~nm}$ domains in our system to be between $3 \times 10^{-3}$ and $1.5 \times 10^{-1} \mu \mathrm{m}^{2} / \mathrm{sec}$. These values differ from those extrapolated from single molecule measurements using the Saffman-Delbrück equation [1]. Given the suggestion that single molecule measurements do not probe the continuum limit, it may be more valid to estimate raft diffusion coefficients by extrapolating down from large domains rather than up from single molecules.

Our analysis of the culled data set in Figure 3 shows that high viscosity membranes produce data that fall well below the dashed line in Figure 3 and that fit Eq. 1 reasonably well. We conclude that any remaining unplotted data that fall well below the dashed line should also fit

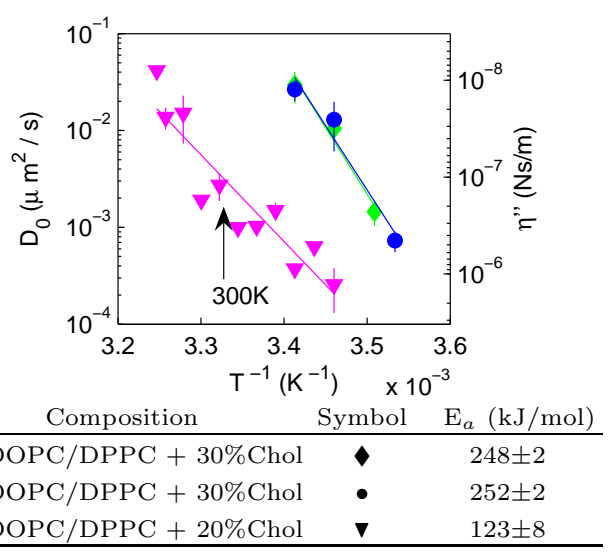

FIG. 4: The diffusion coefficient $D_{0}$ and the 2D membrane viscosity $\eta^{\prime \prime}$ as a function of temperature in membranes with majority $\mathrm{L}_{o}$ and $\mathrm{S}_{o}-\mathrm{L}_{o}$ phases. Solid lines are fit to $D_{0} \sim$ $\exp \left(-E_{a} / k_{B} T\right)$, with $E_{a}$ values shown in the table.

Eq. 1. We use this data to yield a size-independent $D_{0}$ using:

$$
\begin{aligned}
D(r) & =D_{0}\left[\log \left(\frac{\lambda_{0}}{r}\right)-0.0772\right], \\
\text { with } D_{0} & =\frac{k_{B} T}{4 \pi \eta^{\prime \prime}} .
\end{aligned}
$$

Figure 4 shows a plot of the resulting $D_{0}$ and 2D membrane viscosity vs. temperature $(\mathrm{T})$. We find that the $\mathrm{L}_{o}$ phase viscosities for membranes of 1:1 DOPC/DPPC + $30 \% \mathrm{Chol}$ and 1:2 DOPC/DPPC $+30 \% \mathrm{Chol}$ are similar, suggesting that $\mathrm{L}_{o}$ viscosities are not highly composition dependent. In contrast, viscosities for membranes of 1:4 DOPC/DPPC $+20 \%$ Chol are much larger, consistent with these membranes falling within the three phase region in Figure 1

At $22^{\circ} \mathrm{C}$, we find $2 \mathrm{D}$ membrane viscosities for the $L_{o}$ phase of $10^{-8} \leq \eta^{\prime \prime}(\mathrm{Ns} / \mathrm{m}) \leq 5 \times 10^{-7}$. Surface shear rheometry finds monolayer viscosities on the order of $10^{-8}$ to $10^{-6}(\mathrm{Ns} / \mathrm{m})$ only in liquid condensed phases [19], which is consistent with tight packing of lipids in bilayer $\mathrm{L}_{o}$ phases. In contrast, the $2 \mathrm{D}$ membrane viscosity of the $\mathrm{L}_{\alpha}$ phase is low, and results in $\mathrm{D} \sim r^{-1}$. In monolayers, the same $\mathrm{D} \sim r^{-1}$ dependence is found for solid domains diffusing across a liquid background [11].

In the membrane literature, a $3 \mathrm{D}$ membrane viscosity, $\eta_{3 D}$, is defined as $\eta_{3 D} \simeq \eta^{\prime \prime} / h$, where $h$ is the bilayer thickness. Assuming $h=3.3 \mathrm{~nm}$, we find $3 \leq \eta_{3 D}$ (Pas) $\leq$ 150 , on the order of [16, 20] or greater than [21] published values for model membranes. However, the relation between $\eta_{3 D}$ and $\eta^{\prime \prime}$ is not exact, because lipids anchored to the interface differ from a thin homogeneous layer. Indeed, the lipid headgroups often determine the membrane viscosity [7]. This is not always appreciated, and may be a source of ambiguity in discussions of the Saffman-Delbrück model [16]. 
Figure 4 demonstrates that domains diffuse in membranes of high viscosity via an activated process. If the $2 \mathrm{D}$ membrane viscosity $\left(\eta^{\prime \prime}\right)$ were independent of $\mathrm{T}$, we would expect $D_{0}(T) \sim T$. Instead, we find a better fit for $\log \left(D_{0}(T)\right) \sim-T^{-1}$, consistent with an activation energy $E_{a}$ for diffusion such that $D_{0} \sim \exp \left(-E_{a} / k_{B} T\right)$. The data in Figure 4 follow Arrhenius behavior even though a gel phase emerges at low temperature for some mixtures. Composition of the $\mathrm{L}_{o}$ phase varies only slightly with temperature [10]. Activation energies for individual lipids [22, 23, 24] have been attributed to the energy required to hop into an available free volume [7, 24. Larger particles such as protein aggregates yield lower apparent activation energies [17]. Fig. 4 lists activation energies for domains diffusing in $\mathrm{L}_{o}$ phases. We find activation energies greater than those reported for single molecules in similar membranes, including DPPC/Chol membranes at high temperature $(30-80 \mathrm{~kJ} / \mathrm{mol})$ [22], as well as $\mathrm{L}_{o}$ lipids in phase separated DOPC/DPPC/Chol membranes at low temperature $(\sim 80 \mathrm{~kJ} / \mathrm{mol})[25]$.

In summary, we present a simple method for quantifying the movement of domains in membranes with coexisting liquid phases. We find that domains diffuse via Brownian motion, and that diffusion rates are described by different models under different experimental conditions. At high temperatures and in membranes with a continuous $\mathrm{L}_{\alpha}$ phase, membrane viscosity is low, diffusion constants are independent of membrane properties, and domains diffuse with a radial dependence of $D \sim 1 / r$. In membranes with a higher viscosity continuous phase, domain movement does depend on membrane physical properties and the radial dependence can be fit by a Saffman-Delbrück model with $D \sim \log (1 / r)$. For these membranes, we determine $2 \mathrm{D}$ viscosities and report activation energies for domain diffusion.

PC was funded by the Oppenheimer Fund, EPSRC, and the Cavendish-KAIST Cooperative Research Program of MoST Korea. SLK acknowledges an NSF CAREER award and a Cottrell Scholar award. SLV acknowledges a grant from the Cancer Research Institute. We thank Imran Hasnain for help with image analysis, and Klaus Gawrisch for helpful conversations.

* veatch@cmdr.ubc.ca

[1] D. V. J. Nicolau, K. Burrage, R. G. Parton, and J. F. Hancock, Mol. Cell Biol. 26, 313 (2006).

[2] D. Meder, M. J. Moreno, P. Verkade, W. L. Vaz, and K. Simons, Proc. Natl. Acad. Sci. USA. 103, 329 (2006).

[3] A. Kusumi and K. Suzuki, Biochim. Biophys. Acta. 1746, 234 (2005).

[4] A. K. Kenworthy, B. J. Nichols, C. L. Remmert, G. M. Hendrix, M. Kumar, J. Zimmerberg, and J. LippincottSchwartz, J. Cell Biol. 165, 735 (2004); Y. Chen, B. Yang, and K. Jacobson, Lipids 39, 1115 (2004); K. Bacia, D. Scherfeld, N. Kahya, and P. Schwille, Biophys. J. 87, 1034 (2004).

[5] P. Saffman and M. Delbrück, Proc. Natl. Acad. Sci. 72, 3111 (1975).

[6] B.D.Hughes, B.A.Pailthorpe, and L.R.White, J. Fluid Mech. 110, 349 (1981).

[7] R. M. Clegg and W. L. C. Vaz, in Progress in ProteinLipid Interactions, edited by Watts and De Pont (Elsevier, 1982).

[8] M. J. Saxton, Biophys. J. 66, 394 (1994).

[9] Quantitative tie-lines are determined by ${ }^{2} \mathrm{H}$ NMR (manuscript in preparation). Fluorescence microscopy phase boundaries differ from those determined by ${ }^{2} \mathrm{H}$ NMR due to the presence of probe lipid.

[10] S.L. Veatch and S.L. Keller, Phys. Rev. Lett. 89, 268101 (2002); S. L. Veatch and S. L. Keller, Biophys. J. 85, 3074 (2003); S. L. Veatch and S. L. Keller, Biochim. Biophys. Acta. 1746, 172 (2005).

[11] J. F. Klingler and H. M. McConnell, J. Phys. Chem. 97, 6096 (1993).

[12] H.A. Stone and A. Ajdari, J. Fluid Mech. 369, 151 (1998); A.J. Levine, T.B. Liverpool, and F.C. MacKintosh, Phys. Rev. Lett. 93, 038102 (2004); ibid, Phys. Rev. E 69, 021503 (2004).

[13] M. I. Angelova, S. Soleau, P. Meleard, J. F. Faucon, and P. Bothorel, Progr. Colloid Polym. Sci. 89, 127 (1992).

[14] S. L. Veatch, I. V. Polozov, K. Gawrisch, and S. L. Keller, Biophys. J. 86, 2910 (2004).

[15] J. C. Crocker and D. G. Grier, J. Coll. Int. Sci. 179, 298 (1996); We track only features identified on the first frame of a set of images. We do not need to track "new" features because movie segments are short and features in $2 \mathrm{D}$ remain in the field of view. In contrast, colloidal particles in 3D can leave the field of focus.

[16] Y. Gambin, R. Lopez-Esparza, M. Reffay, E. Sierecki, N. S. Gov, M. Genest, R. S. Hodges, and W. Urbach, Proc. Natl. Acad. Sci. 103, 2098 (2006).

[17] C. Lee, M. Revington, S. Dunn, and N. Petersen, Biophys. J. 84, 1756 (2003).

[18] S. J. Plowman, C. Muncke, R. G. Parton, and J. F. Hancock, Proc. Nat. Acad. Sci. 102, 15500 (2005). A. Pralle, P. Keller, E. L. Florin, K. Simons, and J. K. Horber, J. Cell Biol. 148, 997 (2000). P. Sharma, R. Varma, R. C. Sarasij, Ira, K. Gousset, G. Krishnamoorthy, M. Rao, and S. Mayor, Cell 116, 577 (2004).

[19] C. F. Brooks, G. G. Fuller, C. W. Curtis, and C. R. Robertson, Langmuir 15, 2450 (1999).

[20] W. Vaz, J. Stmpel, D. Hallman, A. Gambacorta, and M. D. Rosa, Eur. Biophys. J. 15, 111 (1987).

[21] R. Peters and R. Cherry, Proc. Natl. Acad. Sci. USA. 14, 4317 (1982); C. Chang, H. Takeuchi, T. Ito, K. Machida, and S. Ohnishi, J. Biochem. 90, 997 (1981).

[22] H. A. Scheidt, D. Huster, and K. Gawrisch, Biophys. J. 89, 2504 (2005).

[23] A. Filippov, G. Ordd, and G. Lindblom, Biophys. J. 84, 3079 (2003).

[24] P. F. F. Almeida, W. L. C. Vaz, and T. E. Thompson, Biochemistry 31, 6739 (1992).

[25] G. Oradd, P. W. Westerman, and L. G., Biophys. J. 89, 315 (2005). 